NASA/TM—2006-214396

\title{
Variable Frequency Diverter Actuation for Flow Control
}

Dennis E. Culley

Glenn Research Center, Cleveland, Ohio 


\section{NASA STI Program . . . in Profile}

Since its founding, NASA has been dedicated to the advancement of aeronautics and space science. The NASA Scientific and Technical Information (STI) program plays a key part in helping NASA maintain this important role.

The NASA STI Program operates under the auspices of the Agency Chief Information Officer. It collects, organizes, provides for archiving, and disseminates NASA's STI. The NASA STI program provides access to the NASA Aeronautics and Space Database and its public interface, the NASA Technical Reports Server, thus providing one of the largest collections of aeronautical and space science STI in the world. Results are published in both non-NASA channels and by NASA in the NASA STI Report Series, which includes the following report types:

- TECHNICAL PUBLICATION. Reports of completed research or a major significant phase of research that present the results of NASA programs and include extensive data or theoretical analysis. Includes compilations of significant scientific and technical data and information deemed to be of continuing reference value. NASA counterpart of peer-reviewed formal professional papers but has less stringent limitations on manuscript length and extent of graphic presentations.

- TECHNICAL MEMORANDUM. Scientific and technical findings that are preliminary or of specialized interest, e.g., quick release reports, working papers, and bibliographies that contain minimal annotation. Does not contain extensive analysis.

- CONTRACTOR REPORT. Scientific and technical findings by NASA-sponsored contractors and grantees.
- CONFERENCE PUBLICATION. Collected papers from scientific and technical conferences, symposia, seminars, or other meetings sponsored or cosponsored by NASA.

- SPECIAL PUBLICATION. Scientific, technical, or historical information from NASA programs, projects, and missions, often concerned with subjects having substantial public interest.

- TECHNICAL TRANSLATION. Englishlanguage translations of foreign scientific and technical material pertinent to NASA's mission.

Specialized services also include creating custom thesauri, building customized databases, organizing and publishing research results.

For more information about the NASA STI program, see the following:

- Access the NASA STI program home page at http://www.sti.nasa.gov

- E-mail your question via the Internet to help@sti.nasa.gov

- Fax your question to the NASA STI Help Desk at 301-621-0134

- Telephone the NASA STI Help Desk at 301-621-0390

- Write to:

NASA STI Help Desk

NASA Center for AeroSpace Information 7121 Standard Drive Hanover, MD 21076-1320 


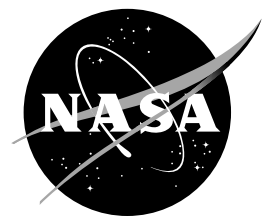

\section{Variable Frequency Diverter Actuation for Flow Control}

Dennis E. Culley

Glenn Research Center, Cleveland, Ohio

Prepared for the

3rd Flow Control Conference

sponsored by the American Institute of Aeronautics and Astronautics

San Francisco, California, June 5-8, 2006

National Aeronautics and

Space Administration

Glenn Research Center

Cleveland, Ohio 44135 


\section{Acknowledgments}

This work was funded under several NASA projects including. Independent Research and Development, Intelligent Propulsion Systems Foundation Technology, and the Subsonic Fixed Wing program.

This report contains preliminary findings, subject to revision as analysis proceeds.

This work was sponsored by the Fundamental Aeronautics Program at the NASA Glenn Research Center.

Level of Review: This material has been technically reviewed by technical management.

Available from

NASA Center for Aerospace Information 7121 Standard Drive

Hanover, MD 21076-1320
National Technical Information Service 5285 Port Royal Road Springfield, VA 22161 


\title{
Variable Frequency Diverter Actuation for Flow Control
}

\author{
Dennis E. Culley \\ National Aeronautics and Space Administration \\ Glenn Research Center \\ Cleveland, Ohio 44135
}

\begin{abstract}
The design and development of an actively controlled fluidic actuator for flow control applications is explored. The basic device, with one input and two output channels, takes advantage of the Coanda effect to force a fluid jet to adhere to one of two axi-symmetric surfaces. The resultant flow is bi-stable, producing a constant flow from one output channel, until a disturbance force applied at the control point causes the flow to switch to the alternate output channel. By properly applying active control the output flows can be manipulated to provide a high degree of modulation over a wide and variable range of frequency and duty cycle. In this study the momentary operative force is applied by small, high speed isolation valves of which several different types are examined. The active fluidic diverter actuator is shown to work in several configurations including that in which the operator valves are referenced to atmosphere as well as to a source common with the power stream.
\end{abstract}

\section{Introduction}

Flow control, broadly defined, is the ability to purposely alter the characteristics of a flowfield in order to extract some overall benefit. More precisely in terms of present day aeronautics technology, flow control implies the intent to correct any local defects in a given flowfield which generally are the result of transitory, three-dimensional effects in a volatile design space. A fixed aeromechanical surface can only be expected to perform optimally over a relatively narrow range of conditions and flow control is viewed as a method in which to extend this useful range. Hence, flow control in aero vehicles and engines is a vigorous area of research. Still the implementation of flow control in actual practice is limited to well-understood and well-behaved techniques such as passive vortex generators and the mechanical articulation of flow surfaces. These passive attempts at flow control are effective and often necessary, however they ultimately limit performance.

NASA Glenn Research Center (GRC) is aggressively pursuing new flow control methodologies and applications in future aero-vehicles. These are largely "active" flow control methodologies meaning that when not needed they are not applied. The intent is to minimize the performance penalty which would otherwise be incurred. In a paper by Lord ${ }^{1}$, et al, many opportunities for active flow control within turbomachinery are articulated. Presently, turbinebased propulsion systems require large aeromechanical design margins and make very limited use of scheduled flow control. The design of these systems is typically based on the worst-case requirements seen at take-off and landing and from the expected performance degradation resulting from routine wear during the service life of the engine. In a typical commercial aircraft, however, only during a small portion of the mission profile will these design limits be approached. The reliance on mechanical margin necessitates a rather severe performance penalty to be incurred during the cruise phase.

Advanced flow control techniques seek to be nonobtrusive, highly responsive, and adaptive. Nonobtrusiveness implies that any performance degradation due to implementation of the technique must be minimal, no matter how it manifests itself in terms of increased weight, power, reduced efficiency, reliability etc. Highly responsive and adaptive control is necessary to enable new aerodynamic designs which lessen the dependency on mechanical margin thereby reducing total system weight and increasing performance.

One key component in the development of active flow control for turbomachinery is improved actuation technology which is the focus of this paper. Active flow control applications involving injection are perceived to be useful within an engine relative to other known techniques because they offer high control authority. Injectionbased techniques require that a pressure source be available which has the energy and capacity to drive fluid into the aerodynamically weak area. In a turbine engine the source of injection fluid is the compressor. Unfortunately use of 
the highly worked fluid from the compressor incurs a substantial performance penalty and its use must be carefully evaluated when determining the overall benefit of an injection-based flow control technology.

In light of the complexity of performing detailed cycle analysis the simple answer is to use as little of the high pressure fluid as possible. It is recognized that modulation of injected flow, when performed at sufficient frequency, is one method which can be used to achieve mass flow reduction while obtaining the desired aerodynamic effects ${ }^{2}$. Modulated, or unsteady injection, is beneficial in other ways as well. Many aerodynamic problems are the result of pockets of low momentum fluid which create blockage, increase losses, or otherwise disrupt the optimal flow of fluid through the engine or over aerodynamic surfaces in general. The abrupt starting and stopping of the injected flow enhances mixing by the vortical entrainment of fluid. When properly applied in a given situation, the enhanced mixing caused by the modulated fluid injection appears to leverage the existing energy in the main power stream flow yielding additional benefit beyond that existing in the energy of the jet alone ${ }^{3}$.

The obvious additional complexity of unsteady injection techniques is cause for concern and thus drives this effort. However, the need for compact, low power, reliable actuation at high frequency opens a myriad of applications in a turbine engine. These applications range from separation control in the inlet, fan, and compressor to active stall control and possibly even cooling mass flow reduction. Numerous other aerodynamic possibilities have yet to be conceived.

\section{Variable Frequency Fluidic Diverter Actuator Design Concept}

We propose that fluidic diverter devices are inherently well suited as actuators for flow control applications in turbomachinery. This is based on two main observations; 1) the behavior of the diverter is based on simple wall jet attachment which is a function of the geometry, hence the possibility exists for robust operation in hostile environments; 2) a very small control force is used to manipulate a much larger flow from which the actuator control authority is generated, therefore the potential for compact and efficient actuation is high. The objective of this investigation is to explore methods of actively controlling the fluidic diverter device to enable variable frequency and duty cycle operation.

Fluidic devices have been known for some time and many variations on their design exist, most of which are well beyond the scope of this paper. The topic is well documented by Gregory ${ }^{4-5}$ who has investigated using piezobenders to actively control fluidics. Most often, fluidic devices are used to produce a fluid jet with a sweeping sinusoidal motion at the exit. There are two types of fluidic devices, classified as diverters and oscillators, that will function in this manner and it is important to distinguish between them. Diverters operate on the Coanda affect and their output is inherently bi-stable; meaning the output will choose and remain in one of two possible states. Oscillators, in contrast, have an inherently unstable output and will never remain stationary.

In most applications, diverters incorporate a passive feedback technique in which a portion of the output flow from each path is redirected back to its point of control. In this configuration, the device is forced to oscillate over a relatively narrow frequency range determined by the length of the feedback path and the source pressure. Fluidic oscillators, in contrast, use an internal chamber in which unstable recirculation regions disrupt the flow through the device causing it to periodically change direction. For our present application, the diverter is of interest because its bi-stable nature is suitable for variable frequency operation.

In choosing the construction of the fluidic device the aerodynamic applications of interest are considered. The author's previous experience $e^{2-3}$ has demonstrated that impulsive fluid injection, when compared to steady fluid injection, has produced dramatically improved results in separation control applications in the compressor. Additionally there appears to be a correlation of benefit to the degree of modulation of the pulsed flow. Highly modulated fluid is more effective than unsteady injection with a constant bias flow. Less clear are the frequency effects in aerodynamic applications. In any case, the effect of frequency is certainly as variable due to the length scales in potential applications.

Most of the limitations with experimental flow control are due to the deficiencies of the available actuation. In general, control authority is provided by the velocity/momentum and frequency of injected flow. An experimenter is typically forced to choose between actuation at high velocity/momentum and low frequency or visa versa. The ideal actuator would provide high control authority and also be low power and low profile to enable embedded point-of-use applications. The actively controlled fluidic diverter valve can potentially achieve all these objectives. 


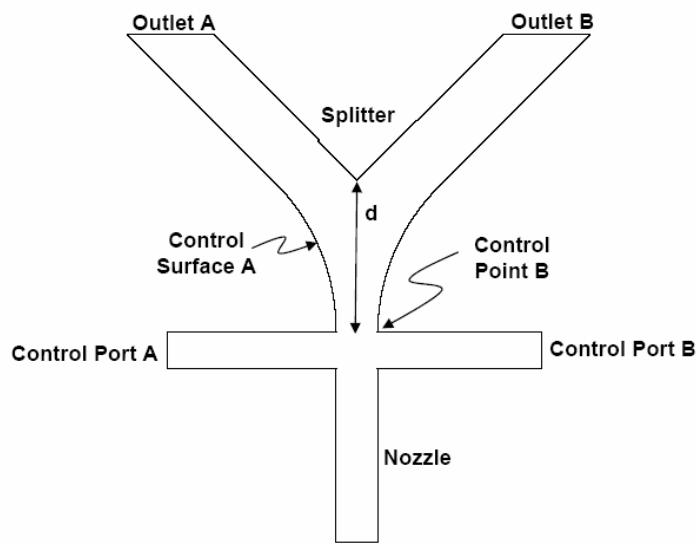

Figure 1 Basic fluidic diverter. A pressurized fluid source at the inlet produces a bi-stable flow which follows a control surface to one of two output ports. By providing an operative force at the appropriate control port a change to the opposite output state can be achieved.
The simplicity of the fluidic diverter valve makes this technology very attractive for embedded flow control applications. In its most basic form, shown in Fig. 1 the diverter valve consists of two convex, axi-symmetric flow surfaces separated by a flow nozzle. Fluid enters the nozzle which serves to develop a highly directional if not laminar flow. Upon exiting the nozzle the fluid jet encounters a highly unstable region in which any small disturbance, due to recirculation or other events, produce a pressure gradient which in turn causes the flow to move toward one flow surface or the other. Once an imbalance develops, the fluid adheres to a wall through the action of the Coanda effect where it travels to the device exit. To achieve the desired pulsed output, the flow at the exit is

segmented into two channels using a splitter. While the jet is very stable following the surface of the wall, only a small amount of force exerted at the appropriate point will cause the flow to detach from the control surface, pass through the unstable region, and adhere to the opposite control surface. This bi-stable nature of the device implies that the switching frequency can be controlled from zero to

a maximum determined by the length scale of the device.

In a typical flow control actuator there is a strong linear relationship of device size and power consumption to the injection velocity/momentum. It is noted that in a diverter a relatively small force is required to disrupt the flow from its stable region causing it to switch to the other side. In effect a small and momentary operative force is leveraged to manipulate the injected power stream flow and should be scalable over a wide range of flow conditions.

In this design concept the frequency and duty cycle of the two output channels are controlled by a pair of active operators which momentarily apply the small force necessary to switch the internal flow paths. The operators used are "high speed" solenoid valves manufactured by three different companies. While they are relatively large with respect to the diverter body, they are intended to gain an understanding of the ideal operator characteristics.

Several recent efforts using fluidic diverter devices have been documented although none directly address frequency capability. Sun ${ }^{6}$, et al, investigated a diverter for combustor applications and note the device's inherent efficiency and robustness to temperature and exit pressure fluctuations. Furthermore they attempt to quantify the necessary control flow to effect switching as a gain, i.e.; outflow to control flow ratio. Brundish ${ }^{7}$, et al, used a diverter for a variable swirl fuel injector and use CFD to predict the control flow to effect switching. Czech and Anderson ${ }^{8}$ propose an interesting concept for noise cancellation by acoustically coupling ambient noise to the diverter control input to produce an amplified output 180 degrees out of phase. Roger and Chan ${ }^{9}$ perform a numerical study of a two-stage fluidic amplifier for exoatmospheric vehicle control.

\section{Experimental Setup}

The experiment attempts to focus on the design and construction of a fluidic diverter actuator for high frequency injection in aero flow control applications. Three fluidic valve bodies are constructed and combined with three pairs of operator valves to produce four test configurations. A method of commanding is developed for precision timing control and the metrics used to evaluate the result are explained.

\section{A. Valve Body and Operator Information}

Three valve bodies, labeled A, B, and C, were constructed using the stereo lithography fabrication process. This rapid prototyping process was chosen because of its low cost, fast turn around, and capability to hold a relatively high dimensional accuracy quoted as $+/-0.076 \mathrm{~mm}(0.003 \mathrm{in})$. Each fluidic valve readily exhibited the bi-stable nature of the diverter configuration when air was applied.

The following narrative describes the features common to each of the three valve bodies. Refer to Fig. 1 for descriptive terms. The construction of each valve body is very simple, consisting of rectangular flow channels sandwiched between two parallel and planar surfaces separated by a height of $1.57 \mathrm{~mm}$ (0.062 in). The remainder of the body is described as two-dimensional and axi-symetric about the nozzle. The nozzle has a square cross section 
and a length-to-width ratio of 5. The two control surfaces are formed using a $12.7 \mathrm{~mm}(0.500 \mathrm{in})$ radius arc tangent to, and extended from, the nozzle over approximately 60 degrees. The control point is defined as the intersection of the nozzle with the control port and is also the point of tangency with the control surface arc. The flow path length from the control point to the fluidic outlet is $38 \mathrm{~mm}$ (1.50 in). The differences between the three bodies are in the control port and exit path dimensions described in the Table 1.

Table 1 Variations in Diverter Valve Body Parameters

\begin{tabular}{|c|c|c|c|}
\hline Valve Body & A & B & C \\
\hline Control port width & $0.79 \mathrm{~mm}(0.031 \mathrm{in})$ & $1.57 \mathrm{~mm}(0.062 \mathrm{in})$ & $1.57 \mathrm{~mm}(0.062 \mathrm{in})$ \\
\hline Control port length & $20 \mathrm{~mm}(0.79 \mathrm{in})$ & $5.08 \mathrm{~mm}(0.2 \mathrm{in})$ & $36.6 \mathrm{~mm}(1.44 \mathrm{in})$ \\
\hline Outlet path width, min & $9.42 \mathrm{~mm}(0.371 \mathrm{in})$ & $9.42 \mathrm{~mm}(0.371 \mathrm{in})$ & $3.15 \mathrm{~mm}(0.124 \mathrm{in})$ \\
\hline $\begin{array}{c}\text { Control point to splitter } \\
\text { distance }\end{array}$ & $18.6 \mathrm{~mm}(0.73 \mathrm{in})$ & $18.6 \mathrm{~mm}(0.73 \mathrm{in})$ & $8.5 \mathrm{~mm}(0.33 \mathrm{in})$ \\
\hline $\begin{array}{c}\text { Minimum nozzle } \\
\text { dimension (actual) }\end{array}$ & $1.57 \mathrm{~mm}(0.062 \mathrm{in})$ & $1.60 \mathrm{~mm}(0.063 \mathrm{in})$ & $1.50 \mathrm{~mm}(0.059 \mathrm{in})$ \\
\hline
\end{tabular}

The valve bodies and operator mounting configurations are shown in Fig. 2 through 5. Valve bodies A and B were specifically constructed for the corresponding operator valves marked X and Y respectively. A single C-body was reconfigured to accept the two different operators $\mathrm{Y}$ and $\mathrm{Z}$. Although the Y-operator is used on two valve bodies they are also installed differently resulting in a difference in the control port path length. Each of the operator valves are commercially available parts and the relevant operator parameters, as provided by the manufacturer, are listed in Table 2. In total there are four actuator configurations using the designations for diverter body and installed actuator. These configurations are labeled AX, BY, CY, and CZ respectively.

Table 2 Operator Parameters and Relevant Mounting Information

\begin{tabular}{|c|c|c|c|}
\hline Operator & $\mathbf{X}$ & $\mathbf{Y}$ & $\mathbf{Z}$ \\
\hline Control port area & $0.79 \times 1.57 \mathrm{~mm}$ & $\begin{array}{c}1.57 \times 1.57 \mathrm{~mm} \\
(0.062 \times 0.062 \mathrm{in})\end{array}$ & $\begin{array}{c}1.57 \times 1.57 \mathrm{~mm} \\
(0.062 \times 0.062 \mathrm{in})\end{array}$ \\
\hline Operating rate & 5 to $10.062 \mathrm{msec}$ & $1000 \mathrm{~Hz}$ & 3 to $5 \mathrm{msec}$ \\
\hline Orifice size & $0.64 \mathrm{~mm}$ & \multirow{2}{*}{$\mathrm{n} / \mathrm{a}$} & $2.95 \mathrm{~mm}$ \\
& $(0.025 \mathrm{in})$ & $0.116 \mathrm{in})$ \\
\hline
\end{tabular}

Figure 6 describes the mass flow characteristics of the three valve bodies when source pressure is applied at the inlet. There was no difference regarding which outlet the flow exited. As previously stated, each nozzle cross section is designed to be identical within the tolerance limits of the fabrication process. Gauge pins were used to determine the minimum nozzle dimension which is listed in Table 1. Using measured mass flow and corrected nozzle cross sectional area, the exit velocity is calculated by the continuity equation, $v=\dot{m} / \rho A$, and is also plotted in Fig 6. Diverter valve body A appears to be slightly high for unknown reasons.

The capability to deliver flow from the operator valves under steady state conditions is also described in Fig 7. In the best case, operator- $\mathrm{Z}$ has a capability of approximately $30 \%$ of the diverter flow. Operators $\mathrm{X}$ and $\mathrm{Y}$ are significantly lower at $5 \%$ of the diverter flow. 


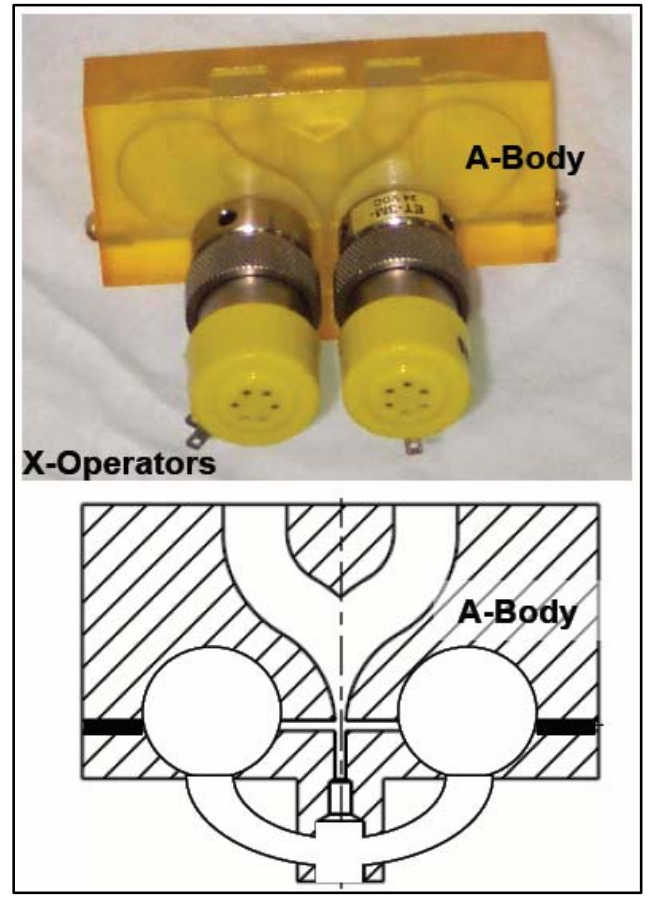

Figure 2. Configuration AX. A photograph of the A-body with X-operators is shown. Below the picture is a cross section of the A-body showing the flow path detail and the use of a common fluid reference for source and control pressure.

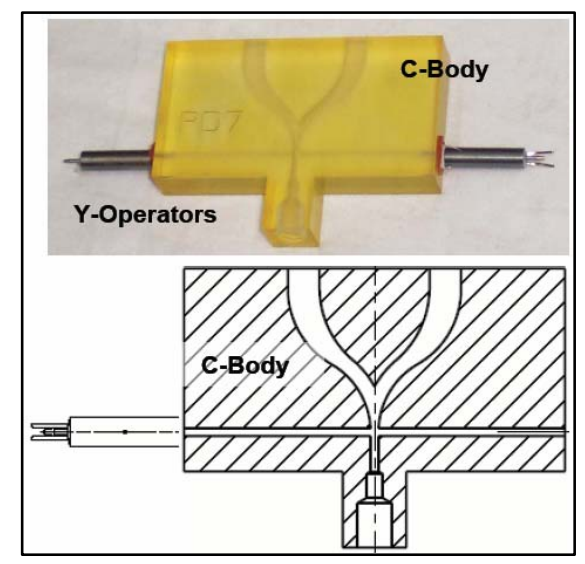

Figure 4. Configuration CY. A photograph of the C-body with Y-operators is shown. Below the picture is a cross section of the $C$ body showing the fluid path detail. The $Y$ operators are mounted externally, one of which is shown at left.

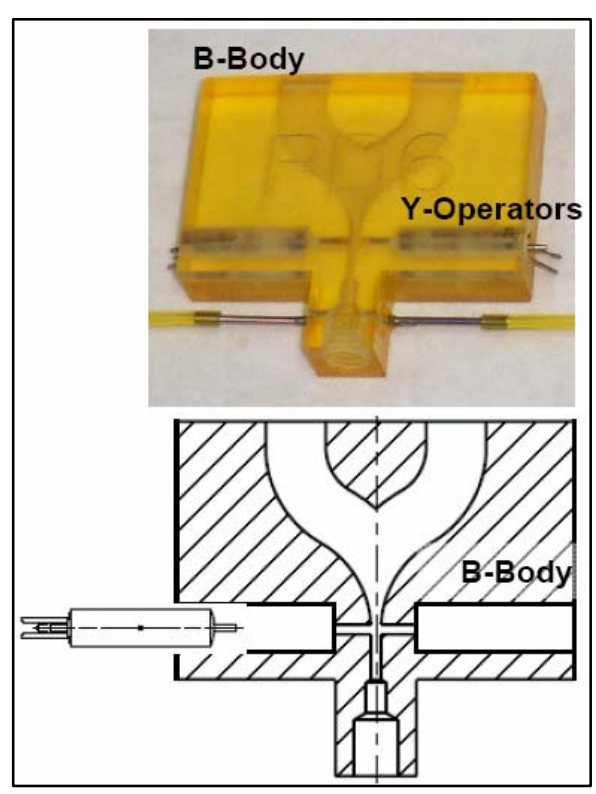

Figure 3. Configuration BY. A photograph of the B-body with embedded Y-operators is shown above. Below the picture is a cross section of the B-body flow path, which is identical to the A-body, except for the operator accommodation. A single $\mathrm{Y}$ operator is shown partially inserted at left.

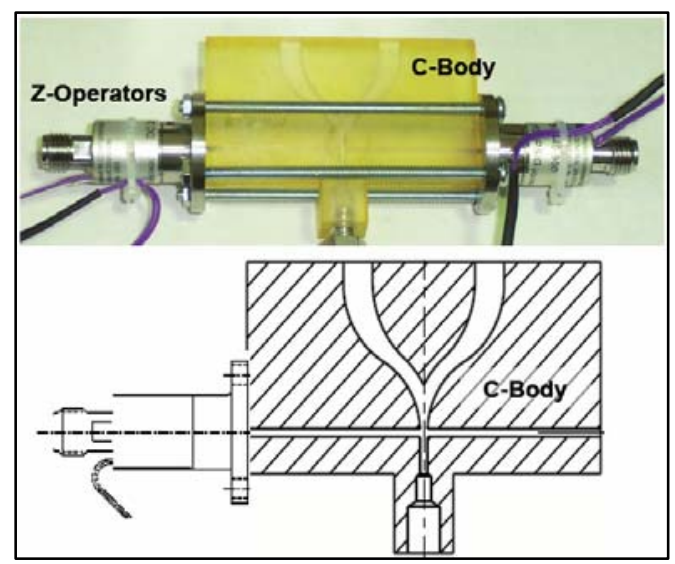

Figure 5. Configuration CZ. A photograph of the C-body with attached Z-operators is shown. Below the picture is a cross section of the C-body flow path. A single Z-operator is shown at left installed on the body. 


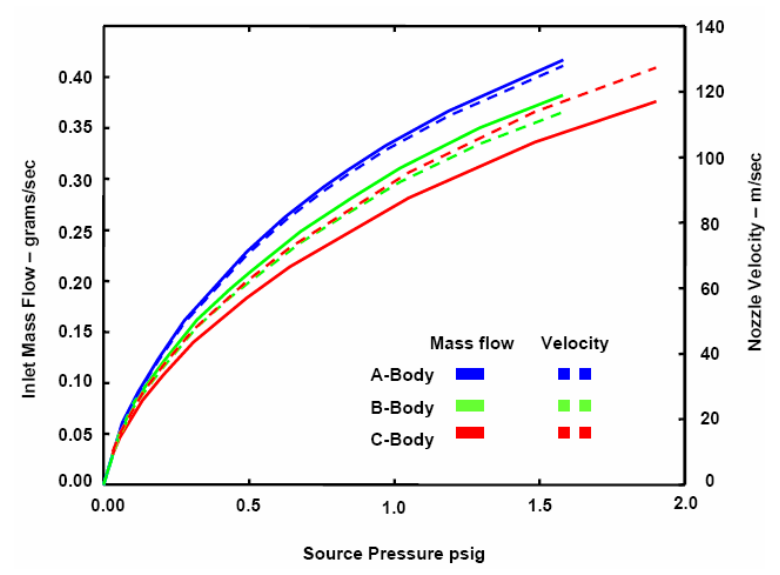

Figure 6. The plot describes the relationship of mass flow to source pressure for the three body configurations used in the diverter. The dashed lines represent nozzle velocity internal to the diverter based on measured mass flow and corrected for actual nozzle dimensions.

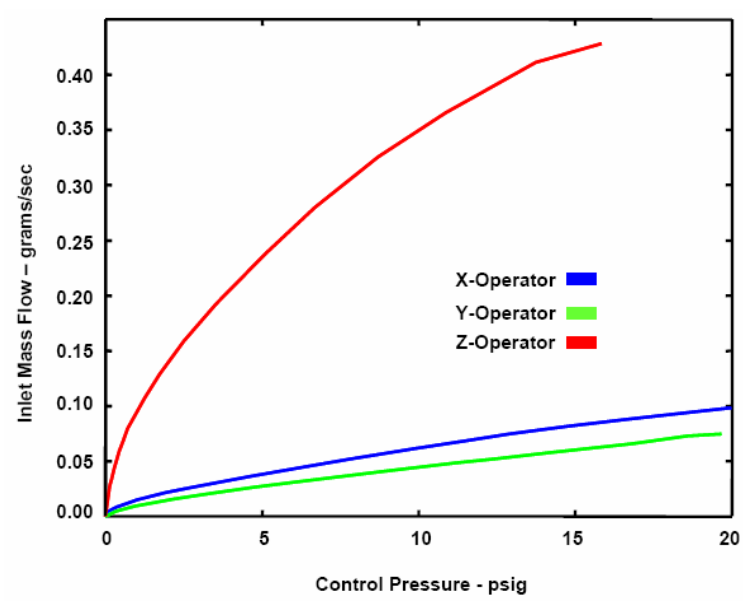

Figure 7. The plot describes the relationship of mass flow to control pressure for the three operator valves used. The data represents a maintained (non-pulsed) state for the valves. The flow capability of the Z-operator is significantly larger than the others.

\section{B. Operator Valve Commanding}

The fluidic diverter is a bi-stable device with two states defined as 'output_A ON/output_B OFF' or 'output_A OFF/output_B ON.' Since it is bi-stable, the control inputs provided by the operator are typically momentary in duration and alternating in sequence. Specifically, an operator action at control port A will cause the flow to turn on at outlet B and off at outlet A as depicted in Fig. 8. An operator action at control port B will result in the opposite state. If the same operator action is applied sequentially it will not result in a change in the flow state. This is exactly equivalent to the operation of an electronic S-R flip flop.

The operating frequency of the diverter valve depends on the frame of reference. When viewed from one of the

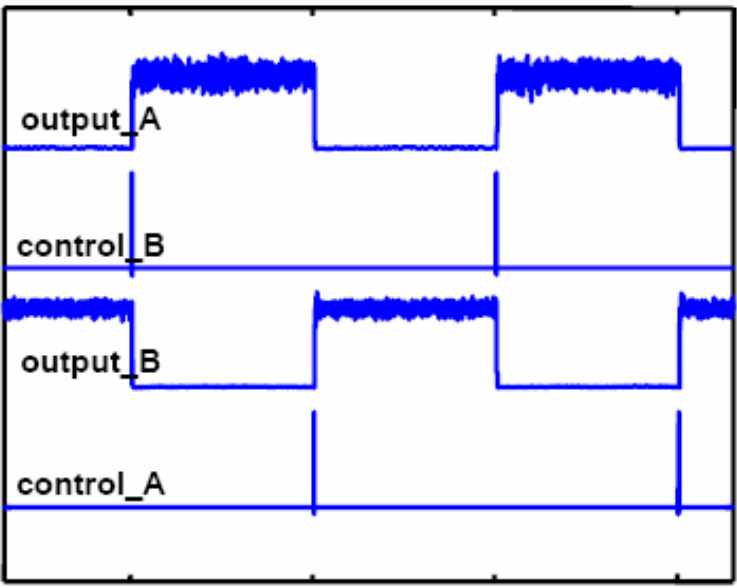

Figure 8. The diverter actuator response to command input is shown. A momentary operator action at control port $B$ results in output A turning on and output B turning off. An operator action at control port $A$ results in the opposite response. pair of outlets, the switching frequency is the same as the operator frequency. When viewed as a pair of outlets the frequency is double since the outlets are switched 180 degrees out of phase. From the inlet frame of reference, the device consumes an essentially constant flow.

Two potential useful characteristics can be derived from the bi-stable nature of the diverter. First, the duration of the momentary action of the operator valve need only be sufficient to insure the diverter changes state. In fact, a longer duration only wastes energy by maintaining the operator valve and disturbing the power stream flow. Secondly, by altering the time relation, or phasing, of the command pulses, the duty cycle of the output flow is modified. Duty cycle is described as the relationship of ontime to the total period. Since there are two outlets of equal and opposite nature, the sum of the duty cycles will always be one.

Referring again to Fig. 8, the time, $\boldsymbol{t}_{\boldsymbol{b - a}}$, refers to the period from the control_B command signal until the next control_A command signal. This is the time period resulting in flow at output_A. The time, $\boldsymbol{t}_{\boldsymbol{a}-\boldsymbol{b}}$, is defined correspondingly and together they define the total period, $\boldsymbol{T}$. Therefore duty cycle is defined as, 


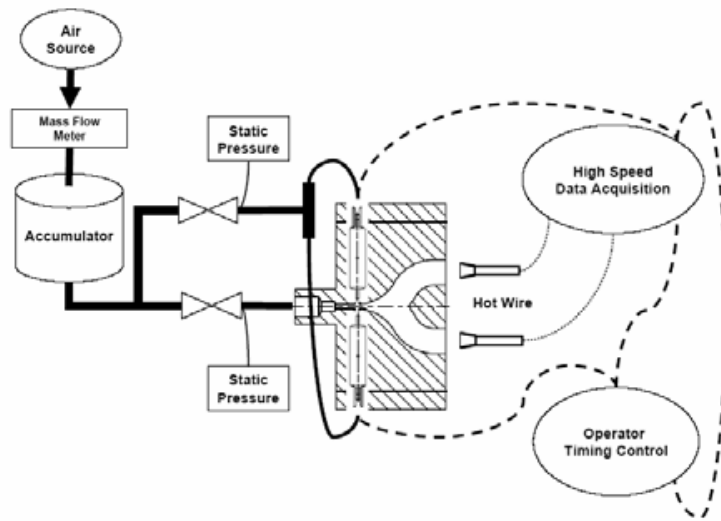

Figure 9. A graphical depiction of the experiment setup is shown. Solid lines indicate air line connections which feed the diverter source and the operator valves. The dashed lines indicate electronic signals for control and data acquisition.

$$
D_{a}=\frac{t_{b-a}}{\left(t_{b-a}+t_{a-b}\right)}=\frac{t_{b-a}}{T}
$$

As long as the operator frequency does not exceed the ultimate diverter response times any frequency or combination of frequencies can be applied.

In order to quantify the effect of operators and diverter configurations, precise timing control is required. Control of the operator on-time is most critical in determining the maximum performance because it enables the determination of the minimum command on-time to achieve switching under all conditions. For operator valves $\mathrm{X}$ and $\mathrm{Y}$ a programmable microcontroller-based drive circuit was constructed which enabled command pulse widths in increments of 0.8 microseconds. The Z-operator valves require higher power and were not compatible with the microcontroller driver. Using a signal generator, a simple digital circuit to provide complimentary drive signals, and a special controller provided by the valve manufacturer, similar timing control was achieved for the Z-operator.

\section{Data Acquisition and Metrics}

The entire experiment setup is graphically depicted in Fig 9. Filtered shop air is used as the fluid source for the experiments. A mass flow meter, 0.006 grams/sec accuracy, is used to measure the average total air flow to the diverter and operator valves. Following the mass flow meter is an accumulator volume to stabilize the pressure source and minimize any acoustic or other feedback noise from contaminating the mass flow measurement. Two pressure regulators downstream of the accumulator are used to independently set the pressure for the diverter body and operator valves except in the case of A-Body / X-Operator which share a common pressure source. Average pressure is independently measured at the two regulators to better than 0.003 psig. On the output, side calibrated hot wire probes are used to monitor the velocity waveforms. Electronic valve commands and the hot wire signals are recorded on a high speed data acquisition system sampled at $10 \mathrm{kHz}$.

\section{Results}

Ultimately we are concerned with developing an active fluidic diverter actuator capable of high frequency, high flow modulation capability. This is not an easy task as it involves a complex interplay between source pressure, control pressure, and minimum operator valve on-time. The methodology involved is to choose some reasonable values for source pressure and then try to isolate the effects of control pressure and valve response.

\section{A. Source Pressure}

The effect of source pressure is to establish a flow velocity exiting the nozzle internal to the diverter. Too low of a pressure and the resulting velocity is insufficient to establish the pressure gradient necessary for the Coanda effect to occur. At very low pressures the flow seems to be evenly distributed between the two outputs. As the source pressure increases the resultant mass flow through the nozzle increases. Referring back to Fig. 6, it appears that a source pressure in the range of 0.1 to 0.2 psig established a mass flow of approximately $0.12 \mathrm{grams} / \mathrm{sec}$. This mass flow developed a nozzle velocity of 30 to $40 \mathrm{~m} / \mathrm{sec}$ which was sufficient to cause the jet to adhere to the control surface. The minimum values are difficult to precisely quantify for several reasons. At these low pressures the regulators tend to drift and the velocity detected at the exit is substantially lower due to the diffusing exit passages and turbulence.

When the pressure is increased above the lower threshold no evidence was observed to indicate that the Coanda effect was diminished. The flow could be forced to either channel by pinching off the output and each channel appeared stable. The pressure and flow rates were substantially increased; however, we did not try to establish a maximum flow rate out of safety concerns.

The importance of this parameter is to understand the turndown capability of the diverter actuator. By controlling mass flow through the device it is possible to fine tune the exit velocity thereby providing a control capability beyond the simple binary output modulation. 


\section{B. Control Pressure}

Control pressure refers to the pressure supplying the operator valves. The operators are used to supply the motive force which disturbs the internal diverter flow thus effecting switching. Ideally we could maintain the operator valves in the open state and adjust the pressure to detect the minimum required for switching. Unfortunately, the regulators are not designed for that purpose and the pressure transients that develop during adjustment can produce misleading results. We chose instead to set the control pressure and pulse the operator valves using a reasonable on-time which approaches the steady state operator flow thereby attempting to minimize the effect of operator valve response.

The operator valve flow characteristics vary substantially as seen in Fig. 7 and it is clear that The Z-Operator has far greater capacity to deliver massflow at a given pressure than the $\mathrm{X}$ or Y-Operators. We would therefore expect that the Z-operator would require a lower control pressure than the others to be effective. This does not appear to be true since the required control pressures for the different operator valves did not correlate well with Fig. 7. All seemed to have approximately the same control pressure requirement to effect switching. The control pressure requirement did increase with increasing source pressure but not in a linear fashion; rather the ratio of control pressure to source pressure was reduced at higher source pressures. It is also noted that differences in the control port path length, when comparing configuration BY to CY, did not have any substantial effect on the required control pressure requirement.

There is some evidence to support that too high of a control pressure contributes to unreliable switching. This may be attributed to a very energetic disturbance which distorts the nozzle flow in a more random fashion with unpredictable results.

It is clear that evaluating the effect of a control disturbance under unsteady conditions, such as those that exist when pulsing with an operator valve, is much more complicated than in the steady state. An improved experimental setup would be beneficial. To adequately quantify the pulsed control requirement would require very fine pressure regulation and the ability to independently measure the minute quantity of mass flow required for each pulse. This would allow the quantitative assessment of unsteady "gain" in which the ratio of the output response is compared to the input. As the experiment currently exists only qualitative assessment of observations can be reported.

\section{Commanded On-time}

The last parameter to be varied was the operator valve on-time which is strictly a function of the valve response characteristics. Two flow conditions, referring to source and control pressure settings, were established which produced reliable switching at the outputs using long duration command pulses. The majority of the data was taken under these conditions. Once the source and control pressures were established, the commanded on-time was reduced to determine the minimum on-time in which reliable switching still occurred. With the minimum commanded on-time established, the period between command pulses was reduced to establish a maximum frequency of operation. The plots of the step response to the minimum commanded on-time and the maximum frequency of operation for the four actuator configurations are shown in Fig. 10 through Fig. 17. Since the diverter actuator outputs are complimentary, only one output pair is depicted, the other being negligibly different. Table 3 is used to show the resulting lag, rise, and fall times for the diverter configurations at a source pressure of $1.5 \mathrm{psig}$. This develops a nozzle velocity in the range of $120 \mathrm{~m} / \mathrm{sec}$.

Table 3 Diverter actuator response characteristics

\begin{tabular}{|c|c|c|c|c|}
\hline Actuator & AX & BY & CY & CZ \\
\hline $\begin{array}{c}\text { Command pulse } \\
\text { time, minimum }\end{array}$ & $11.2 \mathrm{msec}$ & $2.4 \mathrm{msec}$ & $2.4 \mathrm{msec}$ & $1 \mathrm{msec}$ \\
\hline Lag time & $15 \mathrm{msec}$ & $5 \mathrm{msec}$ & $4 \mathrm{msec}$ & $3 \mathrm{msec}$ \\
\hline Rise time & $2 \mathrm{msec}$ & $2 \mathrm{msec}$ & $1.8 \mathrm{msec}$ & $1 \mathrm{msec}$ \\
\hline Fall time & $2 \mathrm{msec}$ & $2 \mathrm{msec}$ & $1.8 \mathrm{msec}$ & $1 \mathrm{msec}$ \\
\hline Maximum frequency & $55 \mathrm{~Hz}$ & $125 \mathrm{~Hz}$ & $125 \mathrm{~Hz}$ & $312 \mathrm{~Hz}$ \\
\hline
\end{tabular}

The path length from the control point to the exit is $38 \mathrm{~mm}$. At the exit, the velocity detected by the hot wire was typically about $35 \mathrm{~m} / \mathrm{sec}$ indicating that the jet was substantially slowed by mixing and diffusion in the passage. Even so the time required to transmit the diverter switching information is at worst 1 msec and probably much less. This indicates the major functional limitation of these actuator configurations is the operator valve because the lag times are all at least three times longer than the transport delay.

The other interesting observation is that the rise and fall times were only marginally affected by the valve response. This seems to suggest that once the disturbance generated at the control point reaches some threshold 


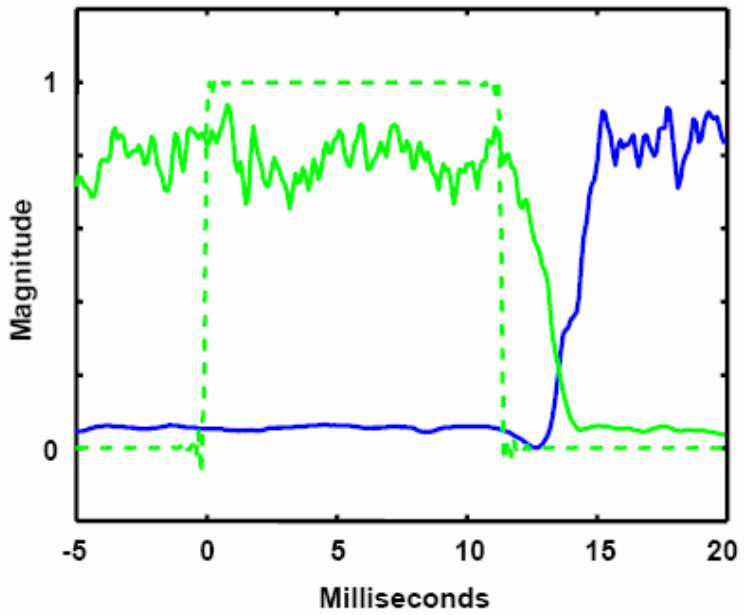

Figure 10. Step function response plot for the A-body fluidic actuator with the $X$ operator. The command pulse width (dashed) is 11.2 msec resulting in a typical lag time of $\mathbf{1 5} \mathbf{~ m s e c}$ and rise/fall times of 2 msec.

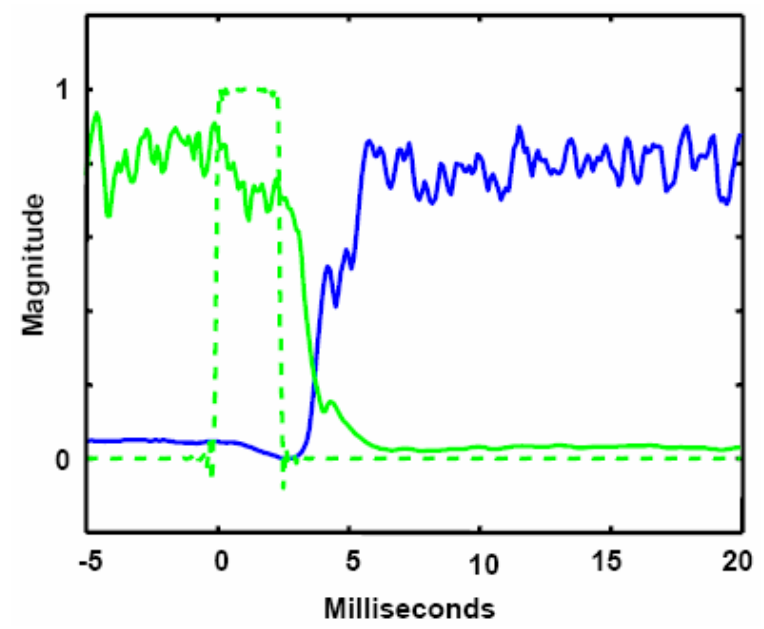

Figure 12. Step function response plot for the B-body fluidic actuator with Y-operator. The command pulse width (dashed) is 2.5 msec resulting in a typical lag time of $5 \mathrm{msec}$ and rise/fall times of 2 msec.

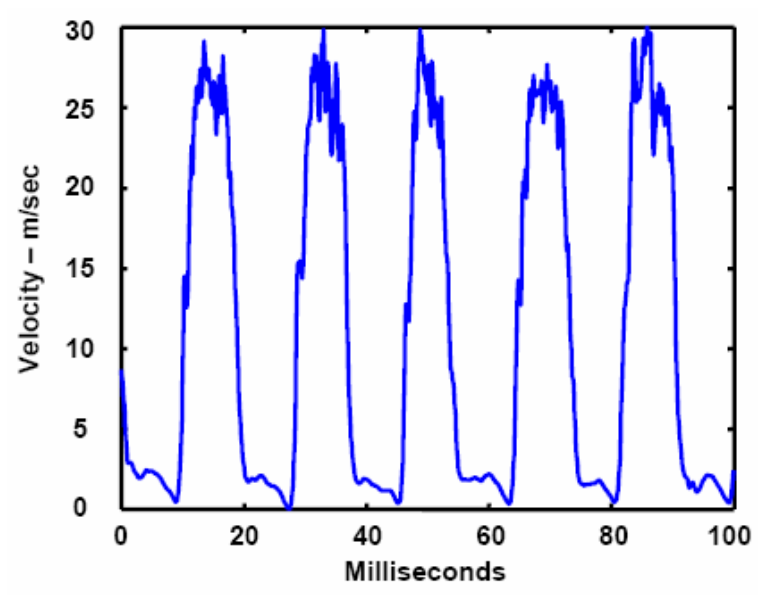

Figure 11. The A-body fluidic actuator with $\mathrm{X}$-operator using a common source and control pressure. Full modulation of the output flow is shown at $55 \mathrm{~Hz}$.

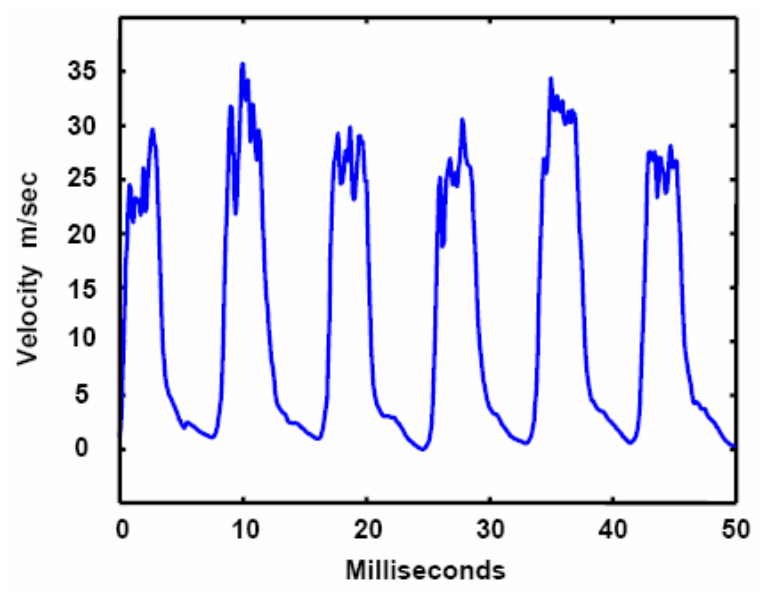

Figure 13. B-body fluidic actuator with Yoperator using separate source and control pressures. Full modulation is shown at $125 \mathrm{~Hz}$.

value the switching occurs. Any disturbance below the threshold value seems to be irrelevant. If this were not true there should be evidence of a longer switch transient especially in the case of the AX actuator configuration.

The concept of a threshold is indicative of an amplifier with a very high gain, where the input signal can quickly saturate the output, and is desired for this application.

\section{Vent Mode Operation}

The last fluidic diverter configuration is a variation on the $\mathrm{CZ}$ actuator except that the control pressure is referenced to the room ( 0 psig). This is shown as an interesting alternative to the previous configurations. The ventreferenced actuator responded to a similar range of source pressures as the $\mathrm{CZ}$ actuator. The dynamic response was significantly different however. In this mode of operation, the commanded operator on-time required a large increase in its duration from $1 \mathrm{msec}$ to at least $9 \mathrm{msec}$. Correspondingly the lag time increased from 3 msec to 6 


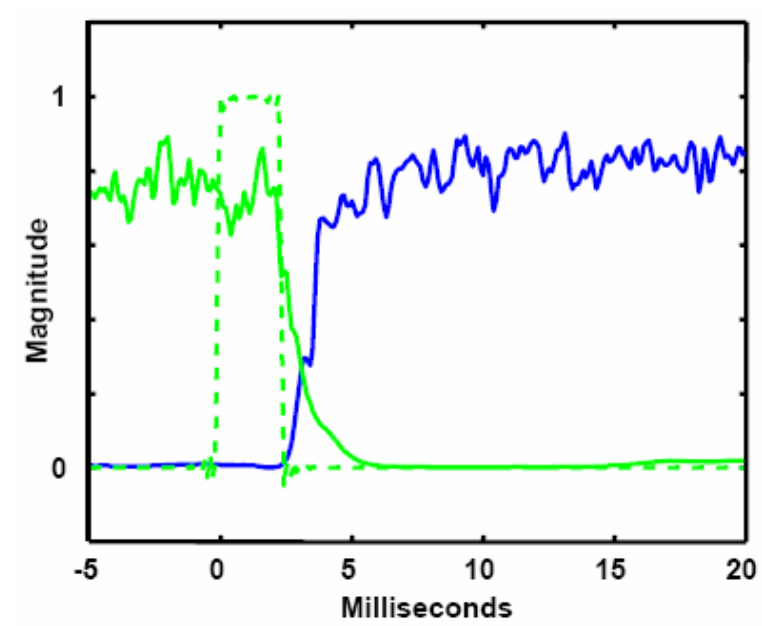

Figure 14. Step response plot for the C-body fluidic actuator with the externally mounted Yoperator. The command pulse width (dashed) is $2.5 \mathrm{msec}$ resulting in a typical $4 \mathrm{msec}$ lag time. The rise/fall times are typically $1.8 \mathrm{msec}$, however the fall time will occasionally persist as shown above.

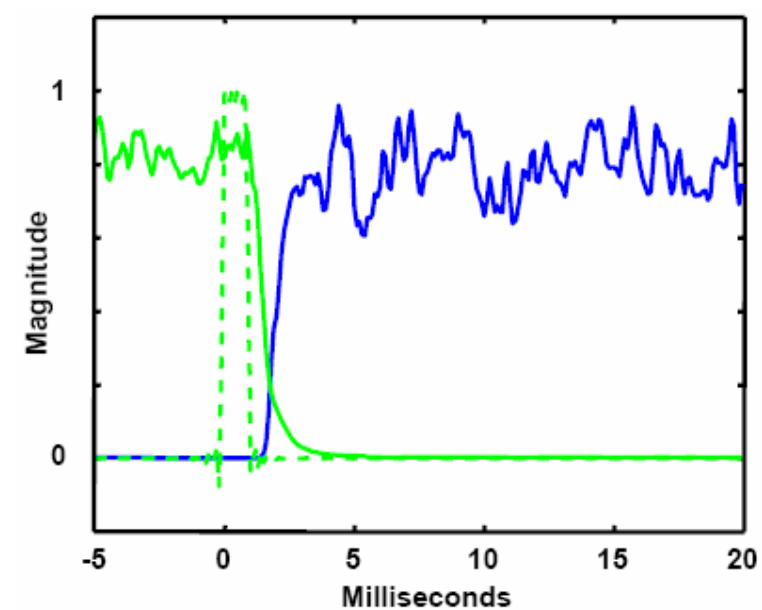

Figure 16. Step response plot for the C-body fluidic actuator with the externally mounted Zoperator. The command pulse width (dashed) is $1.0 \mathrm{msec}$ resulting in a typical $3 \mathrm{msec}$ lag time. The rise/fall times are typically $1 \mathrm{msec}$.

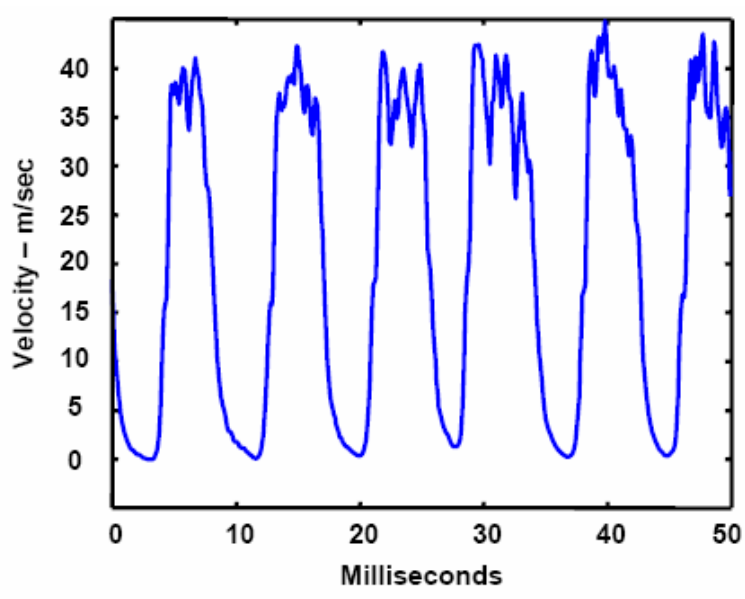

Figure 15. C-body fluidic actuator with Yoperator shown operating with full modulation at $125 \mathrm{~Hz}$.

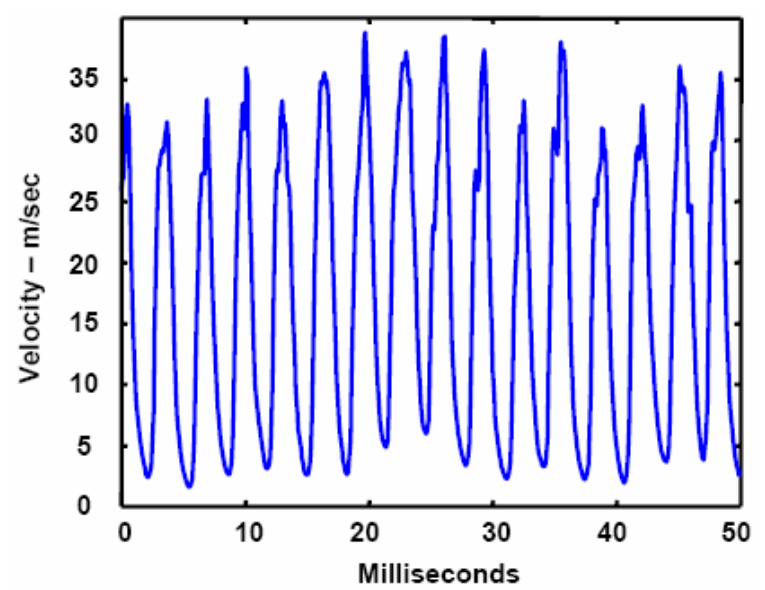

Figure 17. C-body fluidic actuator with Z-operator shown operating with nearly full modulation at 312Hz.

msec. Lower source pressures increased the lag time because the suction was correspondingly reduced. This effect is not a change in the response of the operator valve, since it is the same valve; rather it is a change in the efficiency in which the disturbance mass flow is transported to the control point. Because there is high velocity flow in the nozzle, the static pressure at the control point is lower than the ambient pressure to which the valve is referenced. Just like a pressurized control source the disturbance mass flow is directed into the nozzle. Because it relies on suction, however, it is less efficient and therefore slows the diverter response.

Referring to Fig. 18, this configuration is found to be highly unusual in that the lag time is actually less than the commanded on-time. Shortening the on-time resulted in the actuator not reliably switching. No explanation has been found for this behavior. Fig. 19 shows the maximum frequency operation of $50 \mathrm{~Hz}$ for the vented configuration. 


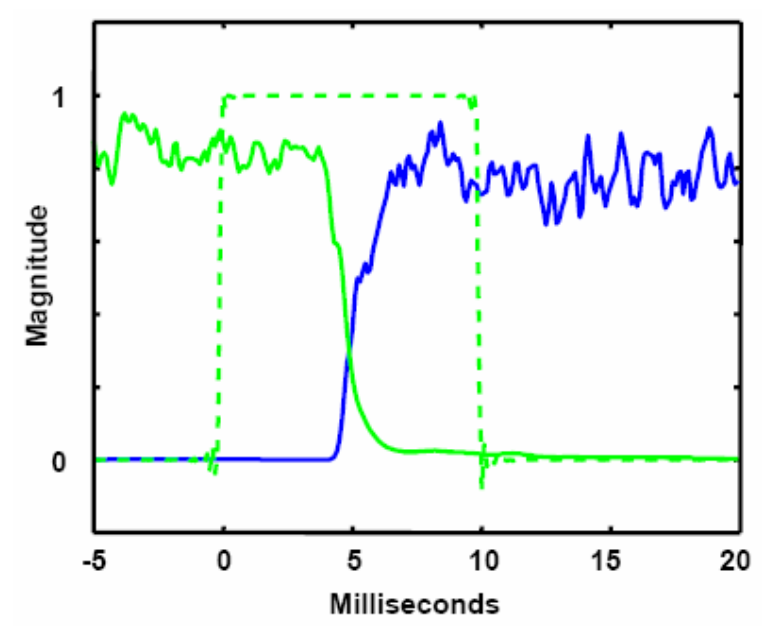

Figure 18. Step response plot for the C-body fluidic actuator with the externally mounted Zoperator in vent mode. The command pulse width (dashed) is $10 \mathrm{msec}$ resulting in a typical $6 \mathrm{msec}$ lag time. The rise/fall times are tvpically 2 msec.

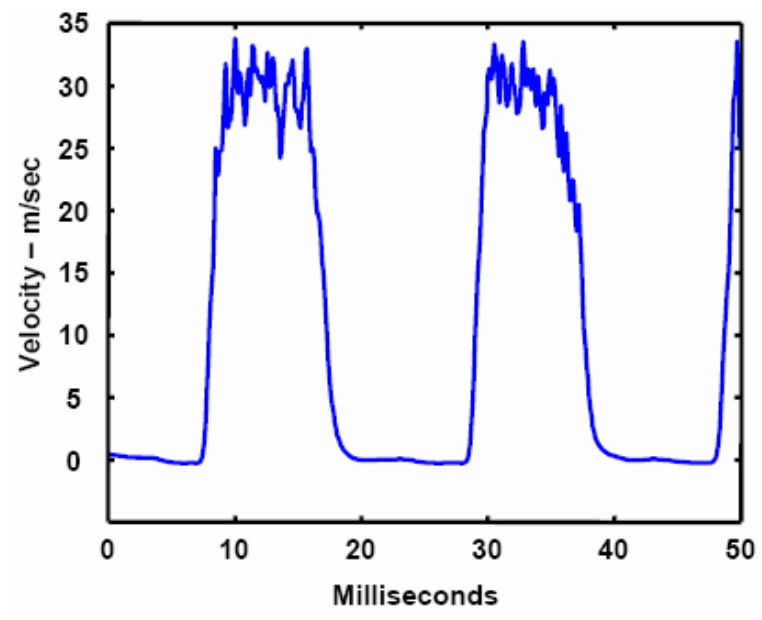

Figure 19. C-body fluidic actuator with Z-operator in vent mode shown operating with nearly full modulation at $50 \mathrm{~Hz}$.

\section{E. Discussion}

In the most basic sense, the objective of demonstrating an actively controlled fluidic diverter actuator was successfully demonstrated. However, to be a viable actuator for most flow control applications there needs to be substantial improvement in frequency response. Passive (those with integral feedback) fluidic diverter oscillators are known to operate at frequencies on the order of $10 \mathrm{kHz}$. These are also the potentially required operational frequencies for high speed turbomachinery applications.

Obviously the limitations in the active configurations are due to the operator, in this case solenoid valves. Surprisingly, however, the highest speed solenoid valve did not provide the highest performance fluidic diverter. This discrepancy points out the need for a better understanding of the forces needed to generate a disturbance which is sufficient to separate the attached flow within the device. Another obvious need is to understand the optimal location for placement of the control point. It would be serendipitous that the location chosen for this experiment was optimal.

Another concern is a reliance for the diverter performance on an operator technology which is itself limited. No solenoid technology is known to have a frequency capability sufficient to achieve $10 \mathrm{kHz}$ operation. Even piezo technology, while probably a better choice, will have a limitation based on the mass, both mechanical and fluid, which must be moved to effect a change in the power stream flow pattern.

The author suggests that a more promising technology to pursue is based on an all-electronic actuation mechanism. This could be plasma or spark. This is the only technology which avoids the displacement of any mass except for that which is already in the power stream.

\section{Conclusion}

The operation of the bi-stable fluidic diverter as an actively driven actuator for flow control applications is shown. The device diverts all the input flow to one of two output channels until forced to a new state where all of the output flow switches to the other output channel. Several different configurations are described in which solenoid valves are used as the operative component actively controlling the valve switching frequency and duty cycle.

The choice of the active operative component is shown to have a substantial impact on the switching bandwidth and responsiveness of the diverter. The operator valve speed alone is not sufficient to achieve the maximum capability of the diverter. The operator valve itself must have enough flow capability to create an aerodynamic disturbance at a point on the Coanda control surface which is sufficient in magnitude to cause a flow separation. Once separated the device flow will find the other stable output flow state. An attempt is made to quantify the performance envelope of the fluidic diverter configurations. 
The results of the experimental investigation are far from conclusive about the design of the optimal fluidic diverter configuration. In fact, it points out the need for additional understanding of the location of the optimal control point on the Coanda surface. It is also suggested that other means of applying an active operative force, such as plasma or electric spark which inherently have higher bandwidth, are more suited to achieving the goal of an embeddable, actively controlled fluidic diverter for injection-based in flow control applications.

\section{References}

${ }^{1}$ Lord, W.K., MacMartin, D.G., Tillman, T.G., “Flow Control Opportunities in Gas Turbine Engines,” AIAA2000-2234, Fluids 2000, Denver, CO, 2000

${ }^{2}$ Culley, D.E., Bright, M.M., Prahst, P.S., Strazisar, A.J., “Active Flow Separation Control of a Stator Vane Using Surface Injection in a Multistage Compressor Experiment,” Journal of Turbomachinery, Transactions of the ASME, Vol. 126, January 2004, pp. 24-34.

${ }^{3}$ Wundrow, D.W., Braunscheidel, E.P., Culley, D.E., Bright, M.M, “Separation Control in a Multistage Compressor Using Impulsive Surface Injection,” NASA TM214361, 2006

${ }^{4}$ Gregory, J.W., Gnanamanickam, E.P., Sullivan, J.P., Raghu, S., "Variable-Frequency Fluidic Oscillator Driven by Piezoelectric Devices,” AIAA 2005-0108, 43 ${ }^{\text {rd }}$ AIAA Aerospace Sciences Meeting and Exhibit, Reno, Nevada, 2005

${ }^{5}$ Gregory, J.W., "Development of Fluidic Oscillators as Flow Control Actuators,” A Thesis Submitted to the Faculty of Purdue University by In Partial Fulfillment of the Requirements for the Degree of Doctor of Philosophy, August 2005

${ }^{6}$ Sun, F., Lin, R., Haas, M., “Air Flow Control by Fluidic Diverter for Low NOX Jet Engine Combustion,” AIAA 2002-2945, $1^{\text {st }}$ Flow Control Conference, St. Louis, MO, 2002

${ }^{7}$ Brundish, K.D., Tippetts, J.R., Woolhouse, R.J., Wilson, C.W., "Initial Optimisation of a Fluidically Controlled Variable Swirl Fuel Injector,” AIAA 1999-2403, 35 ${ }^{\text {th }}$ AIAA Joint Propulsion Conference, Los Angeles, CA, 1999

${ }^{8}$ Czech, M., "Fluidic Wallpaper: An Active Control Concept for Low Frequency Broadband Noise,” AIAA 2003-3189, 9 AIAA/CEAS Aeroacoustics Conference and Exhibit, Hilton Head, South Carolina, 2003

${ }^{9}$ Roger, R.P., Chan, S.C., “Numerical Study of Fluidic Bistable Amplifiers,” AIAA 2003-3459, 33 ${ }^{\text {rd }}$ AIAA Fluid Dynamics Conference and Exhibit, Orlando, FL, 2003 


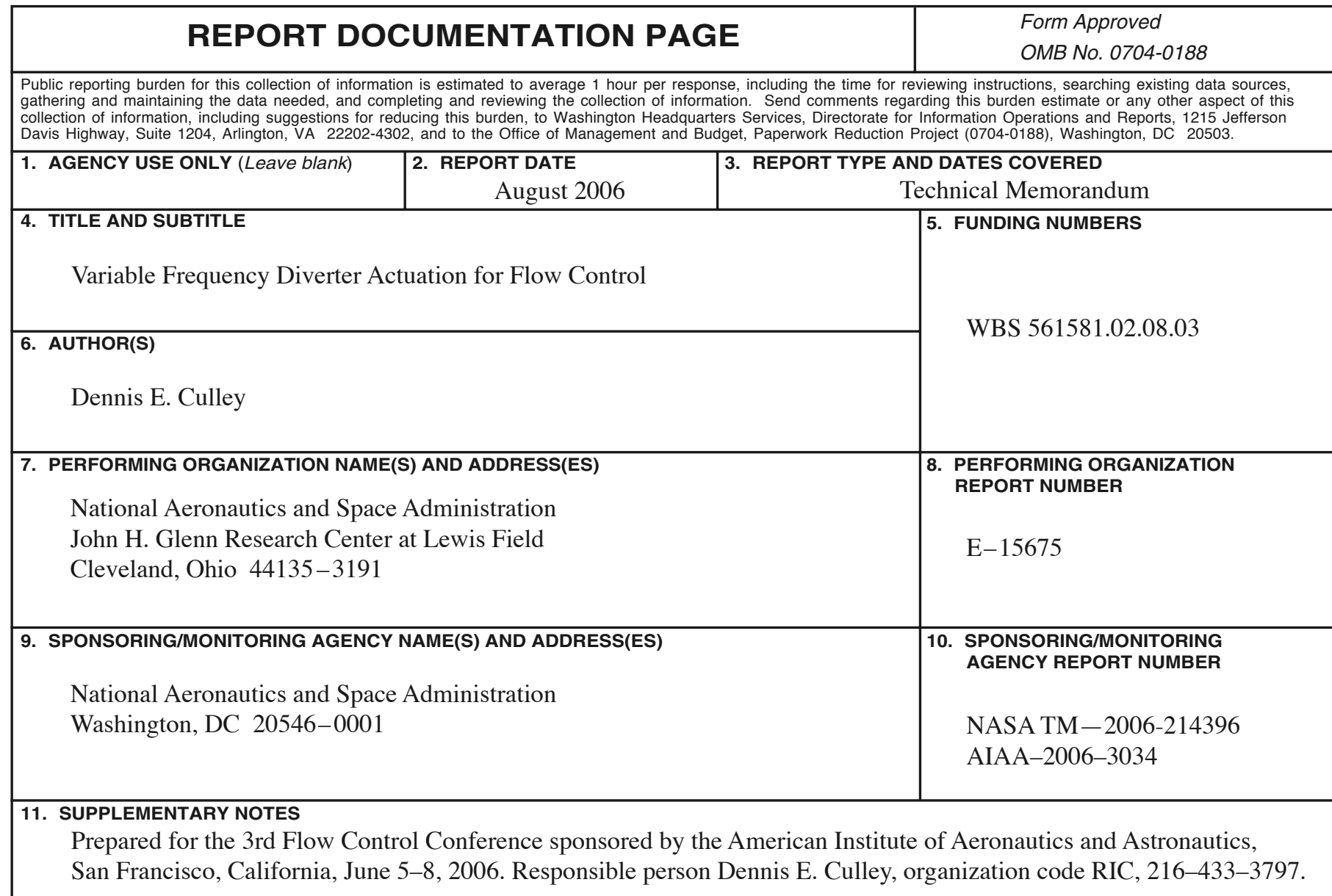

\begin{tabular}{|l|l}
\hline 12a. DISTRIBUTION/AVAILABILITY STATEMENT & 12b. DISTRIBUTION CODE \\
Unclassified - Unlimited & \\
Subject Categories: 34,02 , and 07 & \\
Available electronically at http://gltrs.grc.nasa.gov & \\
This publication is available from the NASA Center for AeroSpace Information, 301-621-0390. & \\
\hline
\end{tabular}

13. ABSTRACT (Maximum 200 words)

The design and development of an actively controlled fluidic actuator for flow control applications is explored. The basic device, with one input and two output channels, takes advantage of the Coanda effect to force a fluid jet to adhere to one of two axi-symmetric surfaces. The resultant flow is bi-stable, producing a constant flow from one output channel, until a disturbance force applied at the control point causes the flow to switch to the alternate output channel. By properly applying active control the output flows can be manipulated to provide a high degree of modulation over a wide and variable range of frequency and duty cycle. In this study the momentary operative force is applied by small, high speed isolation valves of which several different types are examined. The active fluidic diverter actuator is shown to work in several configurations including that in which the operator valves are referenced to atmosphere as well as to a source common with the power stream.

\begin{tabular}{|c|c|c|c|}
\hline 14. SUBJECT TERMS & \multicolumn{2}{|c|}{ 14. SUBJECT TERMS } & $\begin{array}{c}\text { 15. NUMBER OF PAGES } \\
18 \\
\end{array}$ \\
\hline NSN 7540-01-280-5500 & & & $\begin{array}{l}\text { andard Form } 298 \text { (Rev. 2-89) } \\
\text { scribed by ANSI Std. Z39-18 } \\
3-102\end{array}$ \\
\hline
\end{tabular}



